\title{
LOCALITY-PRESERVING NONNEGATIVE MATRIX FACTORIZATION FOR HYPERSPECTRAL IMAGE CLASSIFICATION
}

\author{
Wei $\mathrm{Li}^{1}$, Saurabh Prasad ${ }^{2}$, James E. Fowler ${ }^{1}$, Minshan Cui ${ }^{2}$ \\ ${ }^{1}$ Department of Electrical and Computer Engineering, Mississippi State University \\ ${ }^{2}$ Department of Electrical and Computer Engineering, University of Houston
}

\begin{abstract}
Feature extraction based on nonnegative matrix factorization is considered for hyperspectral image classification. One shortcoming of most remote-sensing data is low spatial resolution, which causes a pixel to be mixed with several pure spectral signatures, or endmembers. To counter this effect, locality-preserving nonnegative matrix factorization is employed in order to extract an endmembers-based feature representation as well as to preserve the intrinsic geometric structure of hyperspectral data. Subsequently, a Gaussian mixture model classifier is employed in the induced-feature subspace. Experimental results demonstrate that the proposed classification system significantly outperforms traditional approaches even in instances of limited training data and severe pixel mixing.
\end{abstract}

Index Terms - Linear mixing model, nonnegative matrix factorization, feature extraction, pattern classification.

\section{INTRODUCTION}

Over the last two decades, land-cover hyperspectral image (HSI) classification [1] has been an important application of hyperspectral data analysis. Hyperspectral imagery contains a wealth of spectral information over a wide range of the electromagnetic spectrum. Compared to the rich spectral resolution, however, the spatial resolution of HSI is relatively poor, especially for high-altitude sensors. For the low-spatialresolution scenario, several land-cover materials often lie in the same pixel; i.e., the spectral values of one pixel are usually mixtures of several pure spectral signatures, also called endmembers [2]. In classification tasks, we often extract features from mixed pixels, although we need to assign a class label to each pixel. Consequently, unmixing is a challenging problem for hyperspectral data analysis. If we assume that the hyperspectral pixel is linearly mixed, the goal of unmixing is to decompose the pixel into a linear combination of pure spectral signatures and corresponding abundance-fraction weights.

Nonnegative matrix factorization (NMF) [3] is a popular blind-source-separation (BSS) [4] method for hyperspectral unmixing. NMF decomposes the data into two nonnegative matrices. As it allows only additive operations, NMF differs from other feature-extraction techniques, such as principal component analysis (PCA) [5] and locality preserving projection (LPP) [6]. In the present work, NMF is employed to extract features for hyperspectral image classification. Specifically, we extract an endmembers-based feature representation from mixed pixels.

As an extension of NMF, locality-preserving NMF (LPNMF) was presented in [7]. LPNMF combines the advantages of NMF and LPP, which leads to a parts-based representation using only additive operations wherein the intrinsic geometric structure is also preserved in the embedded space. In this paper, we also employ LPNMF to extract features for hyperspectral data. A Gaussian-mixture-model (GMM) [8] classifier is used for learning in both the NMF- and LPNMFinduced feature spaces.

The remainder of this paper is organized as follows. In Sec. 2, we briefly review the NMF and LPNMF techniques; in Sec. 3, we introduce the proposed hyperspectral image classification framework using NMF/LPNMF as feature extraction as well as GMM as the classifier. In Sec. 4, we describe the datasets and the classification setup employed as well as experimentally compare the performance of the proposed methods with conventional classification techniques. We finally make several concluding remarks in Sec. 5.

\section{FEATURE EXTRACTION}

\subsection{NMF}

Given a hyperspectral dataset $\mathbf{X}=\left\{x_{i, j}\right\} \in \mathbb{R}^{d \times n}$ with spectral dimension $d$ and $n$ pixels, NMF seeks to find two nonnegative matrices such that $\mathbf{X} \approx \mathbf{U V}^{\top}$, where $\mathbf{U}$ and $\mathbf{V}$ have dimensionality $d \times t$ and $n \times t$, respectively. To solve the NMF problem, Lee and Seung [3] proposed a multiplicative update algorithm:

$$
\begin{aligned}
& u_{i, q} \leftarrow u_{i, q} \frac{\sum_{j}\left(x_{i, j} v_{j, q}\right) / \sum_{q}\left(u_{i, q} v_{j, q}\right)}{\sum_{j} v_{j, q}}, \\
& v_{j, q} \leftarrow v_{j, q} \frac{\sum_{i}\left(x_{i, j} u_{i, q}\right) / \sum_{q}\left(u_{i, q} v_{j, q}\right)}{\sum_{i} u_{i, q}} .
\end{aligned}
$$


During the iterative procedure, each pixel $\mathbf{x}_{j}$ is approximated as $\mathbf{x}_{j} \approx \sum_{q=1}^{t} \mathbf{u}_{q} v_{j, q}$, where $v_{j, q}$ is the abundance component, and $\mathbf{u}_{q}$ is the weights vector. In practice, we have $t \ll d$, which indicates NMF can be viewed as an unsupervised dimensionality reduction.

NMF produces an approximation to $\mathbf{X}$ in the form of a strictly additive combination of basis vectors, differing from other matrix factorization methods such as PCA and LPP. In order to compare the performance of PCA, LPP, and NMF, a simple 2D synthetic dataset with two classes is employed, as illustrated in Fig. 1. We observe that the projection of PCA is estimated according to the variance-maximizing direction. LPP is superior in terms of preserving local (i.e., geometric) structure. As a consequence, for such data with a multimodal distribution, LPP generally offers superior feature extraction compared to PCA. On the other hand, NMF has the advantage of using only additive operations which leads to a parts-based representation.

\subsection{LPNMF}

For hyperspectral data, due to the combination of high spectral and low spatial resolutions, the spectrum of each pixel may be a mixture of several endmembers. For some applications of HSI, such as classification, we hope to find a lowdimensional subspace which captures both an endmembersbased representation as well as the geometric structure simultaneously. As a consequence, LPNMF [7], which combines NMF and LPP and was originally proposed for document clustering, is anticipated to hold significant potential for this problem.

The objective function of LPNMF is

$$
\mathcal{O}=\sum_{i=1}^{d} \sum_{j=1}^{n}\left(x_{i, j} \log \frac{x_{i, j}}{y_{i, j}}-x_{i, j}+y_{i, j}\right)+\lambda \mathcal{R},
$$

where $\mathbf{Y}=\left\{y_{i, j}\right\}=\mathbf{U V}^{\top}$, and $\lambda$ is the regularization parameter. The first part of (3) is the usual objective function of NMF in the form of Kullback-Leibler divergence between $\mathbf{X}$ and $\mathbf{Y}$. On the other hand, $\mathcal{R}$ is used to enforce a geometriclocality constraint among points in the reduced-dimensional subspace $\mathbf{V}(t \ll d)$; i.e.,

$\mathcal{R}=\frac{1}{2} \sum_{j, s=1}^{n} \sum_{q=1}^{t}\left(v_{j, q} \log \frac{v_{j, q}}{v_{s, q}}-v_{s, q}+v_{s, q} \log \frac{v_{s, q}}{v_{j, q}}\right) \mathbf{W}_{j, s}$

where $\mathbf{W}$ is the edge-weight matrix measuring the distance between points in the original space $\mathbf{X}$. This matrix, defined from the theory of LPP [6], is employed to preserve the intrinsic geometry of the data distribution. The following multiplicative rules are used to minimize the function $\mathcal{O}$ and estimate the matrices $\mathbf{U}$ and $\mathbf{V}$ :

$$
u_{i, q} \leftarrow u_{i, q} \frac{\sum_{j}\left(x_{i, j} v_{j, q}\right) / \sum_{q}\left(u_{i, q} v_{j, q}\right)}{\sum_{j} v_{j, q}},
$$

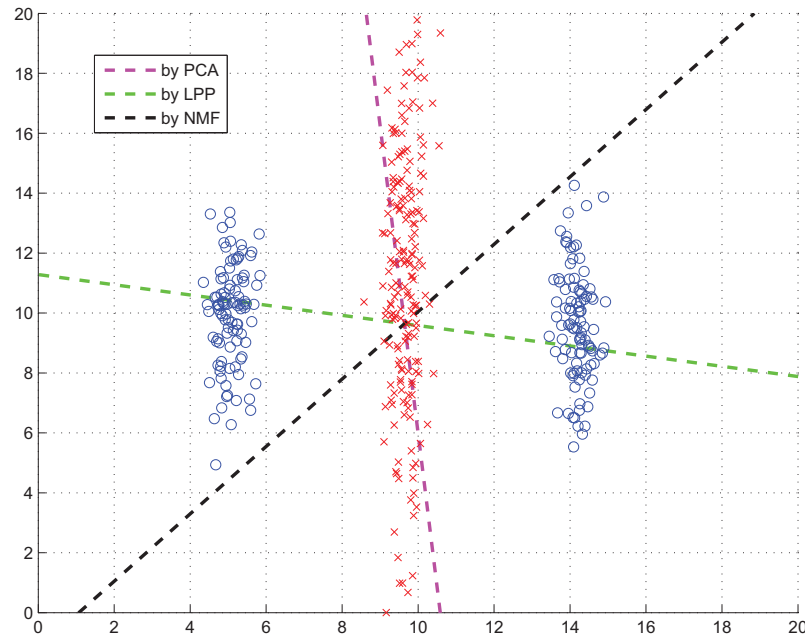

Fig. 1. Example of projections estimated using PCA, LPP, and NMF-a synthetic 2D dataset is embedded onto a $1 \mathrm{D}$ subspace.

$$
\mathbf{v}_{q} \leftarrow\left[\sum_{i} u_{i, q} \mathbf{I}+\lambda \mathbf{L}\right]^{-1}\left[\begin{array}{c}
v_{1, q} \sum_{i} \frac{x_{i, 1} u_{i, q}}{\sum_{q}\left(u_{i, q} v_{1, q}\right)} \\
v_{2, q} \sum_{i} \frac{x_{q, 2} x_{i, q}\left(u_{i, q} v_{2, q}\right)}{\vdots} \\
\vdots \\
v_{n, q} \sum_{i} \frac{x_{i, n} u_{i, q}}{\sum_{q}\left(u_{i, q} v_{n, q}\right)}
\end{array}\right],
$$

where $\mathbf{v}_{k}$ is the $k^{\text {th }}$ column of $\mathbf{V}$, and $\mathbf{I}$ is an $n \times n$ identity matrix. The matrix $\mathbf{L}$ is the graph Laplacian of $\mathbf{W}$. A more detailed description of the LPNMF technique can be found in [7].

Compared to NMF, the locality-preserving constraint of LPNMF guarantees that the local manifold structure is preserved from the original high-dimensional space. This constraint measures the similarity among local neighborhoods via the graph Laplacian which describes the latent distribution structures. For hyperspectral data, the statistical (or geometrical) structure is often complex, such as is the case for multimodal distributions. In [8,9], we illustrated that imposing an additional locality-preserving constraint is important for feature extraction in hyperspectral applications such as classification; such a constraint can provide a tighter distribution and a smaller Bayes error in the resulting feature space.

\section{CLASSIFICATION}

In this work, NMF as well as its LPNMF extension are employed to extract features from hyperspectral data. The motivation for using NMF is the fact that the pixel values of hyperspectral data are nonnegative due to the physics of the sensor. The approximation produced by NMF does not allow negative elements in either the basis vector or the abundance weights. Thus, the representation of hyperspectral data that results from NMF is expected to produce a 
physically meaningful structure. Such an additive representation provides intuitively more meaningful parts-based (i.e., endmembers-based) feature extraction as compared to other approaches which may generate negative elements. LPNMF further incorporates local (i.e., geometric) structure into the representation.

After extracting features using NMF or LPNMF, a GMM classifier is employed. The GMM [8] classifier can be viewed as a combination of two or more normal Gaussian distributions. On one hand, the spectral response of hyperspectral data can be affected by many factors, such as differences in illumination conditions, geometric features of material surfaces, and atmospheric effects. On the other hand, the features extracted by NMF/LPNMF are parts-based (endmembersbased) representations, whose distribution tends to be complex. It is hence reasonable to expect that the statistical distribution for hyperspectral data is a complicated multimodal structure. A classifier such as GMM is hence a natural choose for this work. A detailed description of GMM for classification can be found in [8].

In this paper, we propose a classification of hyperspectral image data which employs NMF/LPNMF for feature extraction, followed by a GMM classifier, the combination of which we refer to as NMF-GMM or LPNMF-GMM. These are compared to a previously developed approach for hyperspectral classification: local Fisher's discriminant analysis (LFDA) followed by GMM classifier [8] (LFDA-GMM). LFDA [8] combines the strengths of linear discriminant analysis (LDA) [5] and LPP, which obtain good between-class separation in the projection while preserving the within-class local structure. Both LFDA and LPNMF employ the locality preserving constraint; however a salient difference between them is that the former is supervised while the latter is unsupervised. To improve the classification performance and to obtain a more intuitive basis, we further exploit LFDA within the LPNMF subspace in order to incorporate the local discriminant constraints within the LPNMF decomposition; the resulting technique is called LPNMF-LFDA-GMM. A similar idea of employing LDA in an NMF subspace was studied for facerecognition tasks in [10].

\section{EXPERIMENTS AND ANALYSIS}

In our experiments, the hyperspectral datasets employed were collected by the Reflective Optics System Imaging Spectrometer (ROSIS) sensor [11]. The images have 115 spectral bands with a spectral coverage from 0.43 to $0.86 \mu \mathrm{m}$, and a spatial resolution of $1.3 \mathrm{~m}$. Two scenes are used in our experiment. The first one is the Pavia city center which has 102 spectral bands with $1,096 \times 715$ pixels formed by combining two separate images representing different areas of the Pavia city. The second scene is the university of Pavia area which has 103 spectral bands with a spatial coverage of $610 \times 340$ pixels. There are nine classes for both datasets. The num- ber of training and testing samples used for the Pavia Centre dataset are 1477 and 8862, respectively, while those for the University of Pavia dataset are 1476 and 7380, respectively.

We evaluate the efficacy of LPNMF for feature extraction followed by a GMM classifier for HSI data. The proposed approaches (LPNMF-GMM, LPNMF-LFDA-GMM) are compared with conventional algorithms, including typical LDA with the quadratic maximum-likelihood-estimation (MLE) [5] classifier (LDA-MLE) as well as the supportvector-machine (SVM) classifier.

Development (tuning) data is derived from the available training data by further dividing training samples into "training" and "testing" samples. As we mentioned previously, NMF as well as LPNMF can be viewed as unsupervised feature extraction, which implies that the reduced dimensionality is an important parameter for NMF-GMM and LPNMF-GMM classification systems. From tuning experiments, the optimal dimensionality of NMF and LPNMF for the Pavia Centre dataset is found to be 20 and 13, respectively; the optimal dimensionalities of NMF and LPNMF for the University of Pavia dataset was 21 and 15, respectively. Since LPNMF-LFDA actually performs two separate dimensionality-reduction processes, we choose the dimensionality of LPNMF to be 15 , and test the optimal dimensionality of LFDA to be approximately 10 according to our experiments. Other details on parameter tuning for SVM and LFDA for these datsaets can be found in [8].

For high-dimensional hyperspectral data, the number of training samples available is often insufficient to estimate the statistical models for each class. In this experiment, we report the classification accuracy of the techniques described above as a function of the training sample size. Figs. 2(a) and (b) illustrate the classification results of the proposed algorithms as a function of the amount of training data employed - the horizontal axis lists the total amount of training samples used. To avoid any bias, the experiments are repeated 20 times, reporting the average classification accuracy. From these results, it can be seen that the proposed LPNMF-GMM and LPNMF-LFDA-GMM approaches outperform other classification techniques. Note that, with decreasing the number of training samples, the overall accuracy for all systems decreases, as expected. However, the rate at which the accuracies for LPNMF-GMM and LPNMFLFDA-GMM drop are much slower than the others. This confirms that proposed methods provide a robust classification performance even with a very small training-data set. Take the University of Pavia dataset for example-when the number of training samples is 590, the overall accuracy of LPNMF-LFDA-GMM is still greater than $90 \%$, which is $4 \%$ higher than that for LFDA-GMM and SVM.

In this experiment, we also compose mixed hyperspectral pixels according to a linear mixing model drawn from background classes which are viewed as "pure" materials. For example, a pixel-mixing abundance of $70 \%$ indicates that $30 \%$ 




(a)

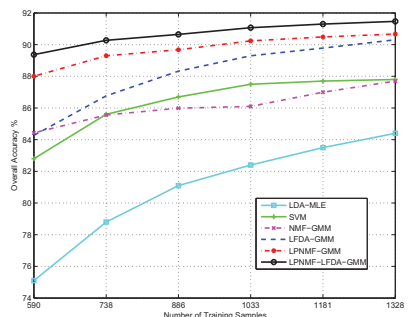

(b)

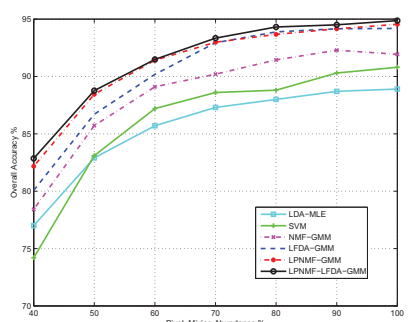

(c)



(d)

Fig. 2. Overall accuracy versus number of training samples for several different classification methods: (a) Pavia Centre and (b) University of Pavia. Overall accuracy versus pixel-mixing abundance: (c) Pavia Centre and (d) University of Pavia.

of background signatures from other classes are mixed linearly with $70 \%$ of the current class. An abundance of $100 \%$ implies that "pure" pixels from the current class are employed without any mixing. Figs. 2(c) and (d) illustrate the overall accuracy as a function of pixel-mixing abundance for the two datasets. NMF-GMM has a comparable classification performance with SVM, both of which yield much better performance than that typical of LDA-MLE. LPNMF-LFDA-GMM has the best performance compared to the other techniques, even under severe pixel-mixing abundance conditions.

\section{CONCLUSIONS}

In this paper, we developed feature-extraction methods based on an NMF for hyperspectral image classification. In the case of hyperspectral data with low spatial resolution, the pixels are typically mixtures of multiple pure endmembers. For classification, this creates a challenge when extracting features. LPNMF, an extension of NMF, reduces the dimensionality of hyperspectral data into a low-dimensional subspace while maintaining both an endmembers-based representation and the geometric structure of the original data. Our classification system employed GMM as the classifier in an LPNMF induced space. Experimental results showed that LPNMF provides effective feature extraction, especially under a mixed-pixel scenario for hyperspectral data. The proposed classification approaches were also shown to outperform others over a wide range of operating conditions, e.g., when very few training samples were employed and under poor pixelmixing conditions.

\section{REFERENCES}

[1] D. A. Landgrebe, "Hyperspectral image data analysis," IEEE Signal Processing Magazine, vol. 19, no. 1, pp. 17-28, January 2002.

[2] A. Villa, J. Chanussot, J. A. Benediktsson, and C. Jutten, "Spectral unmixing for the classification of hyperspectral images at a finer spatial resolution," IEEE Journal of Selected Topics in Signal Processing, vol. 5, no. 20, pp. 521-533, June 2011.

[3] D. D. Lee and H. S. Seung, "Learning the parts of objects by non-negative matrix factorization," Nature, vol. 401, no. 6755, pp. 788-791, October 1999.

[4] S. Jia and Y. Qian, "Constrained nonneagtive matrix factorization for hyperspectral unmixing," IEEE Transactions on Geoscience and Remote Sensing, vol. 47, no. 1, pp. 161-173, January 2009.

[5] R. O. Duda, P. E. Hart, and D. G. Stork, Pattern Classification, 2nd ed. New York: John Wiley \& Sons, Inc., 2001.

[6] X. He and P. Niyogi, "Locality preserving projections," in Advances in Neural Information Processing System, S. Thrun, L. Saul, and B. Schölkopf, Eds. Cambridge, MA: MIT Press, 2004.

[7] D. Cai, X. He, X. Wang, H. Bao, and J. Han, "Locality preserving nonnegative matrix factorization," in Proceedings of the International Joint Conference on Artifical Intelligence, Pasadena, CA, July 2009, pp. 10101015.

[8] W. Li, S. Prasad, J. E. Fowler, and L. M. Bruce, "Locality-preserving dimensionality reduction and classification for hyperspectral image analysis," IEEE Transactions on Geoscience and Remote Sensing, vol. 50, no. 4, pp. 1185-1198, April 2012.

[9] — - "Locality-preserving discriminant analysis in kernel-induced feature spaces for hyperspectral image classification," IEEE Geoscience and Remote Sensing Letters, vol. 8, no. 5, pp. 894-898, September 2011.

[10] S. Zafeiriou, A. Tefas, I. Buciu, and I. Pitas, "Exploiting discriminant information in nonnegative matrix factorization with application to frontal face verification," IEEE Transactions on Neural Networks, vol. 17, no. 3, pp. 683-695, May 2006.

[11] P. Gamba, "A collection of data for urban area characterization," in Proceedings of the International Geoscience and Remote Sensing Symposium, vol. 1, Anchorage, Alaska, September 2004, pp. 69-72. 